\title{
Financiamiento de las empresas hidalguenses
}

\author{
Financing of Hidalgo Companies
}

\author{
Beatriz Sauza-Avila a , Lourdes G. Quiroz-Salas ${ }^{b}$, María M. García-Bravo ${ }^{c}$, Fidencio de la \\ Garza-Herrera ${ }^{d}$, Suly S. Pérez-Castañeda ${ }^{e}$ Dorie Cruz-Ramírez ${ }^{f}$
}

\begin{abstract}
:
One of the main objectives of any company regardlesss of its size is to obtain financial resources to continue growing, having internal or external financing sources that allow them to carry out their activities and thus be competitive in a globalized world, in the This research shows the application of the theory of Pecking Order, considering that for this theory there is no optimal structure of capital when making reference a scale of hierarchies is followed when looking for financing, through this study it will be demonstrated if the theory is applicable to these companies that is to say if they are financed using an order, first with their own resources, secondly external financing, and finally the issuance of shares.
\end{abstract}

Keywords:

Financing, small and medium enterprises

\section{Resumen:}

Uno de los principales objetivos de toda empresa sin importar su tamaño es obtener recursos financieros para seguir creciendo, el contar con fuentes de financiamiento internas o externas que les permiten llevar a cabo sus actividades y de esta manera ser competitivas ante un mundo globalizado, en la presente investigación se muestra la aplicación de la teoría de Pecking Order, considerando que para esta teoría no existe estructura óptima de capital, hace referencia que se debe seguir una escala de jerarquías al momento de buscar financiamiento, a través de este estudio se demostrará si la teoría es aplicable a dichas empresas es decir si se financian utilizando un orden, en primer lugar con sus propios recursos, en segundo lugar financiamiento externo.

\section{Palabras Clave:}

Finanzas, pequeñas y medianas empresas

\section{Introducción}

La teoría del Pecking Orden propuesta por Myers y Majluf (1984), establece que para que las empresas se financien lo pueden hacer en primer lugar a través de autofinanciación, en segundo lugar pueden acceder a contraer deuda y en último lugar optar por emitir nuevas acciones, debiéndose seguir un orden. Para la presente investigación se toma como base 277 empresas hidalguenses del sector turismo, considerándose los

\footnotetext{
"Autor de Correspondencia, Universidad Autónoma del Estado de Hidalgo, Escuela Superior Ciudad Sahagún, Email: Beatriz_sauza@uaeh.edu.mx

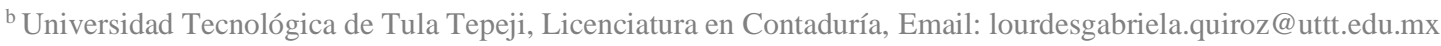

c Universidad Tecnológica de Tula Tepeji, Licenciatura en Contaduría, Email: mariamagdalena.garcia@uttt.edu.mx

${ }^{d}$ Universidad Tecnológica de Tula Tepeji, Licenciatura en Contaduría, Email: fidencio.delagarza@uttt.edu.mx

e Universidad Autónoma del Estado de Hidalgo, Escuela Superior Ciudad Sahagún: sulyp@uaeh.edu.mx

f Universidad Autónoma del Estado de Hidalgo, Escuela Superior Ciudad Sahagún, Email: doriec@uaeh.edu.mx
} 
resultados a través de levantamiento de datos al aplicar un instrumento de medición. Se tiene como objetivo diagnosticar la fuente de financiamiento de las empresas hidalguenses para comparar si cumplen con lo establecido con la teoría del del Pecking Order, y con ello conocer su fuente de financiamiento, para la adecuada toma de decisiones.

El trabajo se encuentra estructurado en seis partes, la primera se define lo relacionado a la estructura de capital, en segundo lugar explicación de la teoría del Pecking Order, en tercer lugar la clasificación de las empresas en México, en cuarto lugar la metodología utilizada, en quinto lugar los resultados obtenidos y por último las conclusiones.

\section{El financiamiento}

De acuerdo a datos obtenidos en la página oficial del Banco Nacional de México (2017) define lo que representa un crédito o financiamiento como es conocido en el ámbito bancario y es:

Un acto a través del cual una persona (acreedor) confía dinero a otra persona (llamada deudor) por un periodo determinado.

Una vez trascurrido el plazo, la persona que recibió el dinero se lo devuelve al acreedor. Usualmente los créditos no son gratuitos, por lo que el deudor, al momento de devolverle el dinero al acreedor o antes, debe agregar un pago adicional o premio al cual se le denomina "interés" y se expresa o se da a conocer a través de la tasa de interés. 1

Así también el término financiamiento se refiere al conjunto de recursos monetario y de crédito que se destinan a una empresa, actividad, organización o individuo, para que los mismos lleven a cabo una determinada actividad o concreten algún proyecto. 6 La financiación se refiere a la combinación de deuda y patrimonio neto pero referido solo a la nueva financiación que se instrumente con alguna de esas fuentes o una combinación de ambas, como pueden ser: ampliar el capital, captar nueva deuda y reducir el reparto de dividendos para favorecer la autofinanciación. 7

El financiamiento se puede obtener internamente mediante la retención de utilidades esto es a corto plazo y a largo plazo se adquieren de manera externa ya sea por la emisión de acciones y bonos en el mercado por medio de mercados financieros que son las instituciones encargadas de reunir a los compradores y vendedores de instrumentos financieros. 8

De acuerdo a la aportación de los diversos autores las empresas pueden estar financiadas de manera interna, de manera externa o una combinación de ambas.

\section{Teoría del Pecking Order}

La teoría de Pecking Order también conocida como la teoría de la jerarquía afirma que no hay estructura óptima de capital, considera que una empresa debe financiarse en orden de elección en fuentes de financiamiento como lo menciona Herrera, de acuerdo a los postulados de la teoría, lo que va a considerar la estructura financiera de las organizaciones es la intención de financiar nuevas inversiones, en primer lugar internamente utilizando los fondos propios, en segundo lugar con deuda de bajo riesgo por ejemplo préstamos bancarios, y en último lugar la emisión de nuevas acciones. 9

Esta teoría de acuerdo con Myers habla de cómo las empresas optan por generar un financiamiento interno, y cuando la misma no cuenta con los recursos adecuados optará por generar una deuda externa, en dado caso que su financiación resulte insuficiente, se emitirán acciones esto ya que al emitir acciones nuevas su valor puede bajar, sin embargo en las grandes empresas funciona mejor la emisión de nuevas acciones ya que cuentan con un cierto prestigio, colocándolas así como principal fuente de financiamiento. 10

En la Teoría del Pecking Order "se establece una cierta ordenanza de preferencias de financiamiento, donde las empresas, en primera instancia se financian mediante recursos propios como beneficios retenidos $y$ aportaciones de los propietarios, en caso de requerir fondos extras, recurrirán a préstamos bancarios y en último lugar a emisión de acciones; el origen de esta preferencia podría ser el costo del financiamiento"

Conforme la teoría de la jerarquía "la estructura de capital de las empresas estaría dictada por una jerarquización que emerge si los costos de emitir instrumentos de financiamiento sobrepasan otros costos y beneficios de dividendos y deuda".

Considerando que la presente investigación se consideraron 277 empresas del sector turismo y por su tamaño en pequeñas y medianas empresas no cuentan con acciones que coticen en la Bolsa Mexicana de Valores, por tal motivo solo se tomará el fundamento de la Teoría del Pecking orden en el primero y segundo orden de su jerarquía es decir si son financiadas con sus recursos propios o bien si han recurrido a financiamiento externo y este último ocurrió una vez que se llevó a cabo el primero. 12

\section{Clasificación de las empresas}

En México algunas de las formas en las que son clasificadas las micro, pequeñas, medianas y grandes empresas, son: por establecimiento, personal ocupado, remuneraciones, producción bruta, activos fijos y número de trabajadores, como se muestra en el gráfico no. 1. 


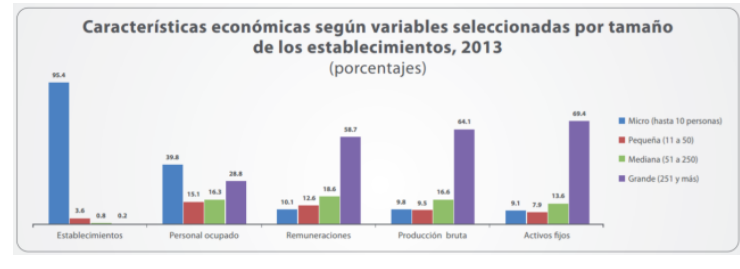

\section{Gráfico 1. Características económicas}

Fuente: INEGI, Censo económico 2014

Las empresas mexicanas que se consideraron para esta investigación pertenecen a la clasificación de las grandes empresas que prestan servicios, de acuerdo a los datos proporcionados por el INEGI los servicios "son todas las actividades económicas que tienen por objeto la satisfacción de un tercero, ya sea en su persona o en sus bienes". 3

En la tabla número 1 se puede observar las micro, pequeñas y medianas empresas, en cuanto a su establecimiento, personal ocupado, remuneraciones, producción bruta y activos fijos. para el tema de estudio que se analizó se tomaron en cuenta las micro y pequeñas empresas del sector turismo del estado de Hidalgo, para conocer cómo se financian, y si estas responden a la teoría del Pecking Order.

Tabla 1. Personal ocupado de las grandes empresas

\begin{tabular}{lrrrr}
\hline \multirow{2}{*}{ Estratos } & \multicolumn{2}{c}{$\begin{array}{c}\text { Personal } \\
\text { ocupado }\end{array}$} & $\begin{array}{c}\text { Crecimiento } \\
\text { porcentual }\end{array}$ \\
\cline { 2 - 3 } Total Servicios & \multicolumn{2}{c}{$\mathbf{2 0 0 8}$} & $\mathbf{2 0 1 3}$ & $\mathbf{2 0 0 8 - 2 0 1 3}$ \\
$\quad$ Micro & $\mathbf{7 3 4 0 2 1 6}$ & $\mathbf{8 1 9 5 0 4 6}$ & $\mathbf{1 1 . 6}$ \\
Pequeños & 3211197 & 3528093 & 9.9 \\
Medianos & 1287862 & 1348207 & 4.7 \\
Grandes & 454721 & 414660 & -8.8 \\
\hline
\end{tabular}

Fuente: INEGI, Censos Económicos 2009 y 2014

\section{Empresas turísticas}

Las empresas turísticas se definen como sociedad $u$ organizaciones estructuradas en variedades de comercio, la cuales tienen como objetivo comercializar servicios profesionales que satisfagan las necesidades del turista, conceptualizado este como toda persona que entra a un lugar distinto de aquel donde tiene fijada su residencia habitual y que permanece en él más de 24 horas y menos de 6 meses con fines de turismo y sin propósito de inmigración. 1, 2

De acuerdo a al SCIAN, la actividad turística se clasifica en:

\section{a. Transporte turístico}

Referido al transporte turístico por tierra, agua o aire con punto de salida y llegada en el mismo sitio.

b. Servicios artísticos y culturales

Referente a producción y prestación de espectáculos artísticos y culturales.

c. Museos, sitios históricos, zoológicos y similares
Dedicadas a exhibir colecciones de carácter artístico, científico, histórico y de valor cultural.

d. Servicios de entretenimiento en instalaciones recreativas y otros servicios recreativos

e. Servicios de alojamiento temporal

Servicio de alojamiento temporal en hoteles, cabañas, villas, campamentos y albergues recreativos.

f. Servicio de preparación de alimentos y bebidas Preparación de alimentos y bebidas para consumo inmediato o para llevar. 3

\section{Metodología de investigación}

Para llevar a cabo el estudio, se estableció un modelo descriptivo, transversal y cualitativo; al identificar las características que tienen las empresas en estudio respecto a las fuentes de financiamiento; transversal al llevarse el levantamiento de campo en un solo momento en el tiempo, que fue diciembre de 2015 y cualitativo, pues la medición de sus conocimientos financieros se hizo a partir de la percepción que tienen los dueños sobre estos temas, a través de una encuesta, integrada en dos apartados: el primero de identificación, con el propósito de conocer las características generales de las empresas y el segundo, sobre temas de educación financiera.

La muestra se calculó a través del muestreo probabilístico estratificado, a partir de la población total de microempresas turísticas en Hidalgo que de acuerdo a INEGI, en 2015, era de mil 728 con más de 5 años de operación, distribuidas con mayor nivel de concentración en los municipios de Huejutla, Pachuca, Tulancingo, Tizayuca, Tula, Inxmilquilpan, Mineral del Chico y Huasca. Analizándose un total de 277 empresas, de las cuales se presentan resultados.

\section{Resultados}

Para conocer si las micros y pequeñas empresas del sector turístico cumplen con lo que establece la teoría del pecking orden se preguntó si los empresarios conocen alguna fuente de financiamiento, contestando el $85 \%$ que no conocen ninguna fuente de financiamiento, en tanto que el $9.75 \%$ si conocen, y el $4.33 \%$ no contestaron, (Tabla 1).

Tabla 1 ¿Conoce alguna fuente de financiamiento para su empresa?

\begin{tabular}{|l|c|c|c|c|}
\hline $\begin{array}{c}\text { Conoce fuente } \\
\text { de } \\
\text { financiamiento }\end{array}$ & Si & No & No contestó & Total \\
\hline Recuento & 27 & 238 & 12 & 277 \\
\hline \% Preferencia & 9.75 & 85.92 & 4.33 & 100 \\
\hline
\end{tabular}

Fuente: Elaboración propia.

Con respecto a los nombres de las fuentes de financiamiento el $2.53 \%$ contestaron relacionado a las 
instituciones bancarias, el $1.44 \%$ solo conocen el financiamiento de Compartamos Banco. (Tabla 2)

Tabla 2 ¿Puede mencionar alguna fuente de financiamiento?

\begin{tabular}{|l|c|c|c|c|}
\hline \multicolumn{1}{|c|}{ Fuente } & Bancos & $\begin{array}{c}\text { Compartamos } \\
\text { Banco }\end{array}$ & No contestó & Total \\
\hline Recuento & 7 & 4 & 266 & 277 \\
\hline \% Preferencia & 2.53 & 1.44 & 96.03 & 100 \\
\hline
\end{tabular}

Fuente: Elaboración propia.

En cuanto a la pregunta relacionada con la obtención de su capital para iniciar su negocio, los empresarios respondieron que el $64.98 \%$ lo hicieron con sus recursos propios, $19.86 \%$ recurrieron a familiares y amigos, $5.78 \%$ micro crédito individual, el $2.53 \%$ utilizó otro, $1.44 \%$ Banca de Desarrollo, y solo el $1.08 \%$ inició con recursos donados, (Tabla 3).

Tabla 3 Para iniciar su empresa, ¿de dónde obtuvo el capital?

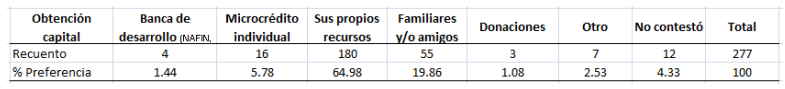

Fuente: Elaboración propia.

Siguiendo el orden de ideas de acuerdo con la tabla 4, las personas utilizaron el dinero de la siguiente manera, el $40.79 \%$ para la instalación de la empresa, $28.16 \%$ producción y venta de productor y/o servicios, $9.75 \%$ no dieron ninguna respuesta, $7.94 \%$ lo utilizaron para otras cosas distintas a lo comprendido en la encuesta, $6.14 \%$ para la elaboración de planes de negocios, 1.44\% invirtió en capacitación. (Tabla 4).

Tabla 4 ¿Para qué utilizó principalmente ese dinero con el que inició su empresa?

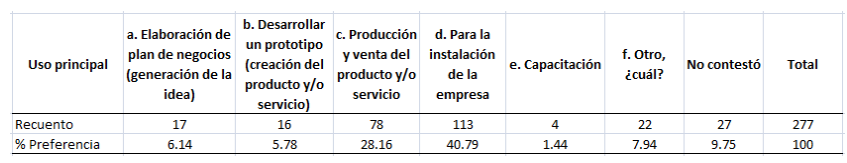

Fuente: Elaboración propia

En lo que corresponde al $5^{\circ}$ año de operaciones de la empresa y si han sido financiadas las repuestas fueron el $80.14 \%$ no han solicitado financiamiento para su empresa $y$ el $12.64 \%$ si lo han solicitado, y solo un $7.22 \%$ no contestaron. (Tabla 5)

Tabla 5 Después del 5 año de operaciones, ¿ha solicitado financiamiento para su empresa?

\begin{tabular}{|l|c|c|c|c|}
\hline $\begin{array}{c}\text { ¿Ha solicitado } \\
\text { financiamiento } \\
\text { para su } \\
\text { empresa? }\end{array}$ & Si & No & No contestó & Total \\
\hline Recuento & 35 & 222 & 20 & 277 \\
\hline \% Preferencia & 12.64 & 80.14 & 7.22 & 100 \\
\hline
\end{tabular}

Fuente: Elaboración propia

En lo relacionado en cuantas veces ha solicitado financiamiento el $80.14 \%$ no han solicitado ninguna vez, el $8.30 \%$ solo una vez, con relación al $2.89 \%$ han solicitado hasta dos veces, $1.44 \%$ hasta tres veces, (Tabla 6).

Tabla 6 Si la respuesta es sí, ¿cuántas veces ha solicitado financiamiento?

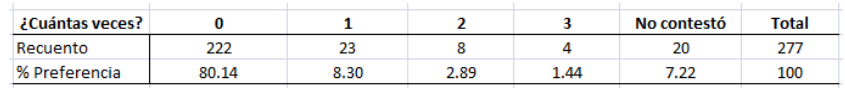

Fuente: Elaboración propia

En cuanto a la causa de porque solicitó financiamiento el $61.73 \%$ no contestó, el $14.44 \%$ lo utilizó para crecer o expandirse, el 14.08 otra causa no considerada en el instrumento de medición, $7.22 \%$ por falta de liquidez para hacer frente a las operaciones diarias, (Tabla 7).

Tabla 7 Primordialmente para qué solicitó esos financiamientos

\begin{tabular}{|c|c|c|c|c|c|c|}
\hline $\begin{array}{c}\begin{array}{c}\text { ¿Para qué } \\
\text { solicitó esos } \\
\text { financiamientos? }\end{array} \\
\text { ? }\end{array}$ & 0 & $\begin{array}{l}\text { a. Por falta de } \\
\text { liquidez para hacer } \\
\text { frente a las } \\
\text { operaciones } \\
\text { diarias }\end{array}$ & $\begin{array}{l}\text { b. Para crecer o } \\
\text { expandirse }\end{array}$ & c. Otro & No contestó & Total \\
\hline Recuento & 7 & 20 & 40 & 39 & 171 & 277 \\
\hline \% Preferencia & 2.53 & 7.22 & 14.44 & 14.08 & 61.73 & 100 \\
\hline
\end{tabular}

Fuente: Elaboración propia

Las principales dificultades que han enfrentado los empresarios para obtener financiamiento son el $38.63 \%$ tamaño de la empresa, $12.27 \%$ información financiera, $7.58 \%$ otro diferente a los considerados en el instrumento, $7.22 \%$ falta de garantías, $6.50 \%$ tamaño de los montos solicitados, $5.05 \%$ falta de formalidad de las empresas, $4.33 \%$ patrimonio de propietario de la empresa, $1.44 \%$ plan de negocios, $1.08 \%$ no solicitó.

Tabla 8 ¿Cuál considera que es la principal dificultad a la que se ha enfrentado para obtener financiamiento externo a sus recursos o a los de sus familiares?

Fuente: Elaboración propia 


\section{Conclusiones}

De acuerdo a los resultados obtenidos se concluye que la investigación cumple con el objetivo al diagnosticar la fuente de financiamiento de las empresas hidalguenses de los municipios de Huejutla, Pachuca, Tulancingo, Tizayuca, Tula, Inxmilquilpan, Mineral del Chico y Huasca. Analizándose un total de 277 empresas, comparando si se cumplen con lo establecido en la teoría del del Pecking Order, y con ello conocer su fuente de financiamiento, para la adecuada toma de decisiones. Se concluye que las micro y pequeñas empresas del sector turismo utilizaron el $64.98 \%$ sus recursos propios para financiarse, siendo un total de 180 empresas, de estas solo 35 de ellas han solicitado financiamiento para su empresa, por tal motivo se deduce que la mayoría de empresas si cumplen con lo establecido con la teoría del peking order al tener un orden para financiarse primero con recursos propios y en segundo lugar acuden al financiamiento externo.

\section{Referencias}

1] Barreto, M. (2007). Turismo y cultura. Pasos: España.

[2] Barreto Mújica, M. (2006). Responsabilidad social. Su inherencia con las micropyme, el turismo y las escuelas universitarias de gerencia. Universidad de Carabobo: Venezuela.

[3] INEGI, 2015, Sistema de clasificación industrial, Recuperado de http://www.inegi.org.mx/est/contenidos/proyectos/SCIAN/presentacio n.aspx

[4] Myers, S.C. \& Majluf, N.S. (1984). Corporate Financing and Investment Decisions when Firms Have Information that Investors Do Not Have. Journal of Fiancial Exonomics, 13(2), p.p. 1-188.

[5] Banco Nacional de México (2017). Sistema Financiero. Recuperado el 17 de abril de 2017 de http://www.banxico.org.mx/divulgacion/sistemafinanciero/sistema-financiero.html.

[6] Definición ABC (2017). Definición de Financiamiento. Recuperado el 18 de abril de 2017, de http://www.definicionabc.com/economia/financiamiento.php

[7] Pérez Carballo, J. (2014). La estructura de capital: cómo financiar la empresa. Madrid: ESIC. p. 35.

[8] Van Horne, J., \& Wachowicz, J. (2002). Fundamentos de administración financiera. México: PERSON EDUCACIÓN. p. 452, 471, 506.

[9] Gutierrez, M., Aguayo, P. \& Panes, J. (2014), Estructura de Financiamiento de las Empresas Chilenas Aplicación del Modelo de Jerarquía, Horizontes Empresariales (1), pp-pp 7-19, recuperado de http://revistas. ubiobio.cl/ index.php/HHEE/ article/view/2397/2099.

[10] Myers, B. (2007). Fundamentos de finanzas corporativas. España: McGRAW-HILL. p. 436
[11] Gómez Martínez, A., García Pérez de Lema, D., \& Marín Hernández, S. (2009). Restricciones a la financiación de la PYME en México: una aproximación empírica. Análisis Económico, XXIV(57), 219-220.

[12] Fama, E., \& French, K. (2002). Explorando teorías de estructura de capital en latinoamérica. Obtenido de Cuadernos de Administración: http://www.redalyc.org/pdf/205/20516983008.pdf 